\title{
A lost cohort of medical students
}

The views expressed in this editorial are those of the author and do not necessarily reflect the position of the Canadian Medical Association or its subsidiaries.

T he intrusive presence of coronavirus disease 2019 (COVID-19) has certainly been felt on many levels. We have been bludgeoned by the brutal social and medical outcomes of not being prepared to deal with this illness. It has changed the way we interact and socialize and even how our family units function. The reality is that not only is this pandemic is not over, it will not end soon even with a vaccine, and there will be much more uncertainty than we want to accept. The pandemic snuck up on the people who were supposed to know what to do - the World Health Organization, the Centers for Disease Control and Prevention, Health Canada, and the provincial health ministries amongst other entities and individuals. This has allowed our next generation of physicians to be hit abnormally hard by this disease and the processes in place (or not in place) to deal with it.

The next generation of medical students will need to be able to deal with future pandemics. They will live in a world of personal protective equipment (PPE) and fluid and aerosol dangers even more than my generation had to. COVID-19 is a deadly pandemic, but one that can be controlled with appropriate low-level PPE and sanitary measures to defend against infectivity. This is not Ebola - but Ebola or its close relative could be coming during the careers of this next generation of health care providers.

Medical education must prepare students and residents to practise in pandemic conditions, and that education should proceed uninterrupted, regardless of a pandemic. This has not been the case in many institutions during COVID-19. The pandemic response has been to keep students at home. Certainly, they have been told to stay home for their protection, and the PPE shortage has had something to do with that decision. But the consequence is that students are being held back. Their elective schedule has been curtailed - not just electives out of the country, but even electives within their own city. Medical specialties with a high barrier to entry require that candidates have several electives to ensure exposure to more than one institute, maximizing the chances of success in a resident match. And that is just for the students who are fortunate enough to already know what specialty they want to practise. What about the students who are undecided and need exposure to several options in order to make a decision that will have such a profound effect on the rest of their lives?
The students are dealing with this lack of access in light of hypocrisy from administration. The Quebec government, for example, asked the students to help in the long-term care centres at the bleakest point - and the students graciously volunteered. No one from administration stepped up and said it was too dangerous to allow despite the well-documented poor conditions and high death rates in long-term care. So why can't the students have their planned education programs back now in a much better-controlled environment?

For our second-year students the all-important transition to clinical practice was cancelled, with the students being sent home for most of their rotation of 24 weeks. Definitively, this subverts the classic patron-to-student teaching model. Some online learning opportunities were formed to address these shortcomings; however, it is during these rotations that students acclimatize to patient care. Online courses that are not validated may not be the best way to teach these crucial first contact moments. While they may be good at modelling some parts of patient interaction, they definitely cannot model the real-life oddities that often happen.

It is not just the first years of clinical training that have been disrupted. The final-year students have had their training cut short as well - but with no back up plan. So, students are graduating having experienced decreased clinical responsibilities and having received limited exposure to different environments that would inform their choice of career and practice of medicine. Not ideal. Another year of medical students is coming on board soon. They are a rare resource and the future of our medical system. Has anything changed to ensure we don't lose this year as well?

\section{Edward J. Harvey, MD}

Affiliation: Co-editor-in-chief, Canadian fournal of Surgery

Competing interests: E.J. Harvey is the chief medical officer of Greybox Solutions, the co-founder and head of medical innovation of NXTSens Inc., the co-founder and chief medical officer of MY01 Inc., and the co-founder and director of Strathera Inc. He receives institutional support from J \& J DePuy Synthes, Stryker and Zimmer, and he is a board member of the Orthopedic Trauma Association and the Canadian Orthopaedic Association. No other competing interests were declared.

DOI: $10.1503 /$ cjs.020620 